\title{
On the possibility of ion-drag to induce dynamic instability in the lower thermosphere neutral gas
}

\author{
C. M. Hall \\ Tromsø Geophysical Observatory, University of Tromsø, Norway \\ e-mail: chris.hall@phys.uit.no
}

Received: 26 January 2000 / Revised: 19 April 2000 / Accepted: 3 May 2000

\begin{abstract}
Strong wind shears may result in dynamic instability, often characterised by the Richardson number lying between zero and 0.25 . The extent to which electric-field driven ion flow may induce such neutral wind shears is examined. Further, it is proposed that, in the ionosphere, it is possible for electric fields to drive ion winds such that the collisionally induced neutral air response may be comparable to viscous damping of neutral motion. We shall present an analogy to the Reynolds Number Re to quantify this effect. In the same way that $R e$ may be used to evaluate the likelihood of a flow being turbulent, the analagous metric may also indicate where in the atmosphere plasma dynamics may be strong enough to destabilise the neutral dynamics.
\end{abstract}

Key words: Ionosphere (auroral ionosphere; ionosphere-atmosphere interactions) - Meteorology and atmospheric dynamics (turbulence)

\section{Introduction}

The turbopause is the height at which turbulent and molecular diffusivities are equal. Danilov et al. (1979) (hereafter referred to as DKP79) obtained estimates of the turbopause altitude at high latitude $\left(80^{\circ} \mathrm{N}\right)$ using a series of rocket soundings over some years. These authors then compared their results with other solarterrestrial metrics, in particular the auroral index $a_{k}$. These results are not presented here; only mention of their finding is made that turbopause height increases with increasing auroral activity. Due to the nature of DKP79, no explanation was proposed for this phenomenon; since $a_{k}$ is derived directly from the departure of the geomagnetic field from its unperturbed value (e.g. Jacobs, 1989), it would seem plausible that the cause lies in the E-region electrodynamics. It is not a foregone conclusion that increases in turbulent intensity at heights below the turbopause will contribute to changes in turbopause height. Indeed Blum and Schuchardt (1978) predicted quite the opposite of the DKP79 result. For the turbopause height to be elevated, the intersection of the turbulent and molecular diffusivity profiles must be elevated (by definition), and one means of achieving this is by enhanced turbulence at the very height of the usual intersection. It is in this height regime that electrodynamics represents an important feature of the atmosphere. We shall quantify the capability of electrodynamics to affect neutral turbulence and, to achieve this end, we propose an "electrodynamics" analogy to the Reynolds Number, Re.

The Reynolds Number $R e=\mathrm{U} L / v$ (where $\mathrm{U}$ is a typical velocity and $L$ is the length scale associated with it and $v$ is the kinematic viscosity, assumed to be equal to the molecular diffusivity). If one thinks in terms of eddies (now known to be a gross oversimplification, if not misconception of real turbulence), eddy velocity and eddy size may be used to estimate $R e$, in which case $R e=$ "eddy" viscosity/kinematic viscosity (e.g. Tennekes and Lumley, 1972). Thus, one could define the turbopause height to be that at which $R e$ is unity. Normally one associates turbulent flows with $R e \gg 1$, but one can reconcile the turbopause height definition by considering that when $R e<1$ turbulent eddies cannot exist because they are damped out by viscosity before, hypothetically, an "air parcel" has sufficient time to complete one "eddy".

\section{Theory}

Overall, the most plausible mechanisms to explain the observations of DKP79 are founded on the concept of auroral activity affecting neutral turbulent intensity as opposed to vice versa. In the mesosphere and lower thermosphere, gravity wave flux and stability may be affected by changes in zonal wind and/or by aurorally induced changes in temperature structure. To change the height of the turbopause $h_{t}$ however, the turbulent 
intensity must change at or very near the nominal turbopause. At such heights, in the lower thermosphere, enhanced ion velocities may be capable of increasing dynamic instability. Dynamic stability is often parametrised by the gradient Richardson number (e.g. Tennekes and Lumley, 1972), Ri, equal to the square of the Brunt-Väisälä frequency divided by the square of the vertical shear of the horizontal wind. It is recognised that the atmosphere is dynamically unstable for $R i<0.25$, whereas if turbulence is already present, it may be maintained by $R i<0.4$ (e.g. Weinstock, 1978). When $R i$ reaches unity however, turbulence ceases (e.g. Roper and Brosnahan, 1997).

At any latitude, atmospheric turbulence is invariably highly layered, inhomogeneous and non-stationary. The same characteristics apply to the ionised component of the atmosphere, particularly at high latitude and during auroral disturbances making it difficult to model the effects of ion-drag. As stated in the Introduction, given $R e=\mathrm{U} L / v$, where $\mathrm{U}$ is a typical velocity, $L$ is a corresponding length scale and $v$ is the kinematic viscosity, it is not uncommon to note that $R e$ is also an approximation to $K / D, K$ being the turbulent diffusivity (e.g. Tennekes and Lumley, 1972) and $D$ is the molecular diffusivity such that $R e=1$ at $h_{t}$. We may model $K$ using for example Hall et al. (1998), who use profile shapes suggested by Shimazaki (1971) scaled to the heights and values of maximum $K$ reported by Danilov and Kalgin (1996). An example is shown in Fig. 1, to portray the nature of both $K$ and $D$ (the latter for three different temperatures to illustrate that it is indeed $K$ that must change in order to affect $h_{t}$ ). Maintaining a constant $D$ profile, we may change $R e$ by either changing the wind speed in the layer defined by $L$ or by changing the layer thickness or both. The results of order of magnitude changes in $R e$ are shown in

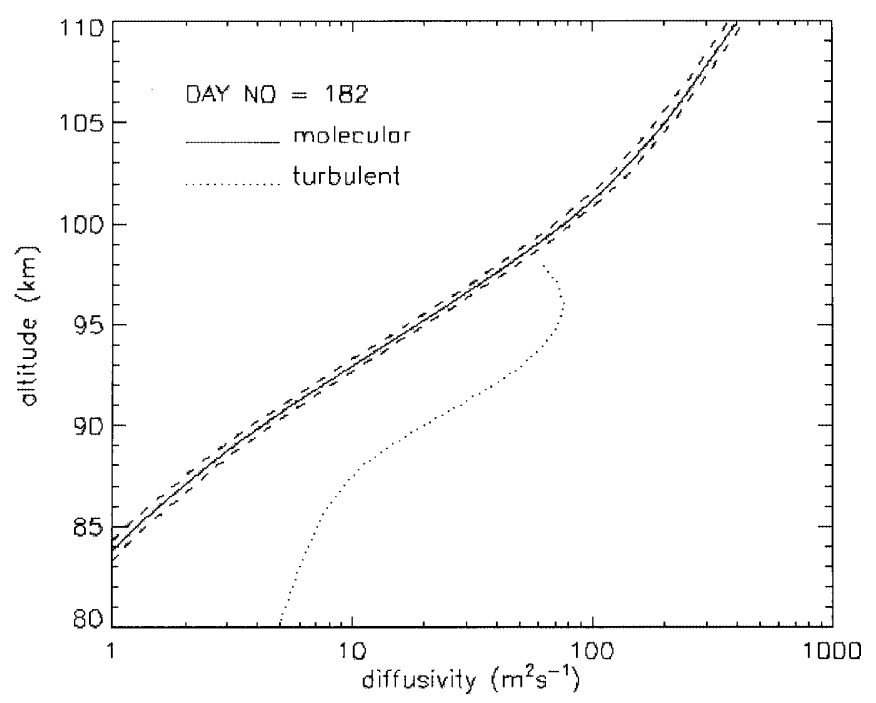

Fig. 1. Effect on turbopause height due to changes in kinematic viscosity induced by changes in temperature. The turbulent diffusivity is modelled by Hall et al. (1998) (although the exact form of the profile is irrelevant here), while the spread in the molecular diffusivity (viscosity) profile represents a $\pm 10 \%$ temperature change
Fig. 2. If we consider the length scale $L$ to be constant we see (from Fig. 2) that even increasing the neutral wind by an order of magnitude will only result in lifting the turbopause by $3 \mathrm{~km}$. Similarly, if neutral velocity changes could be induced over shorter vertical distances (i.e. increasing the wind-shear), say shorter than the typical buoyancy scale length $L_{B}$, i.e. around $200 \mathrm{~m}$ in the lower thermosphere then there would be greater dynamic instability. Evidence of suitable velocity gradients are not easily found in the literature, however, partly due to appropriate height resolution in velocity measurements only being available from in situ methods.

Recent radar-based observations of ion-velocity at the latitude of the turbopause measurements are given by Fujii et al. (1998) and Nozawa and Brekke (1999), the latter for different degrees of solar activity. We shall use Fujii et al. (1998, Eq. 7b) to investigate further the potential ion drag has to either enhance existing turbulence or destabilise the neutral dynamics and thus allow turbulence to exist higher up than usual. Fujii et al. (1998, Eq. 7b) specify the ion motion $\mathbf{V}$ perpendicular to the magnetic field $\mathbf{B}$ to be:

$\mathbf{V}_{\perp}^{2}=\frac{\left[\mathbf{U}_{\perp}^{2}+2 k_{i}\left(\frac{\mathbf{U} \cdot \mathbf{E}}{|\mathbf{B}|}\right)+k_{i}^{2}\left(\frac{|\mathbf{E}|}{|\mathbf{B}|}\right)^{2}\right]}{1+k_{i}^{2}}$

where $\mathbf{U}_{\perp}$ is the neutral wind $\mathbf{U}$ in the same plane, $\mathbf{E}$ is the electric field and $k_{i}$ is the ratio of the ion gyro frequency $\Omega_{i}$ to the ion-neutral collision frequency $v_{i n}$.

Below around $130 \mathrm{~km} k_{i} \ll 1$ so that rearranging Eq. (1) and simplifying

$\mathbf{U}_{\perp} \approx \mathbf{V}_{\perp}-\frac{\Omega_{i}}{v_{\text {in }}} \frac{\mathbf{E}}{|\mathbf{B}|}$

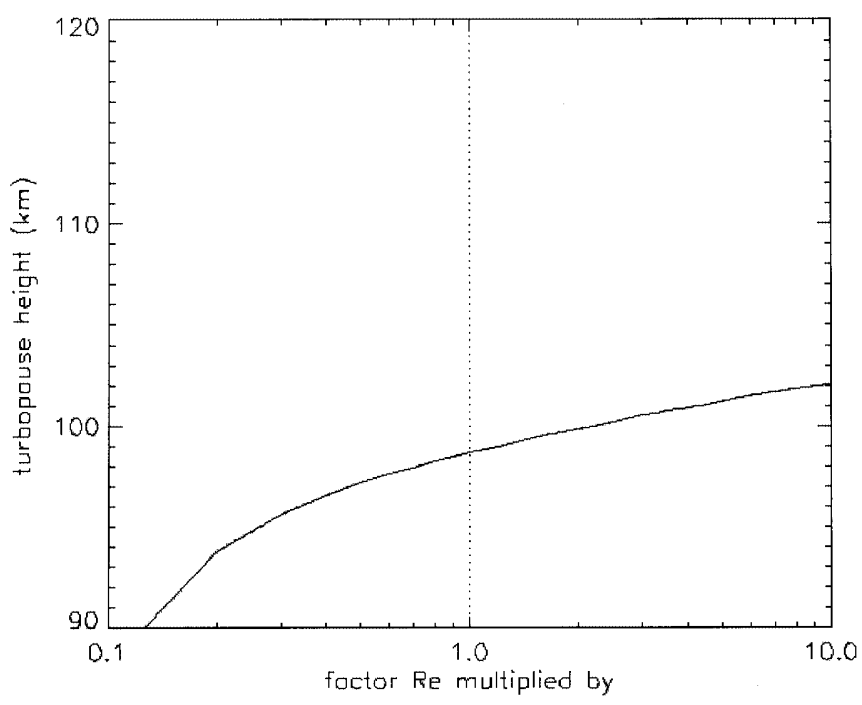

Fig. 2. Change in turbopause height as a result of modifying Reynolds Number $R e$ by the factor on the abscissa. The normal turbopause is where the factor $=1$; multiplying the overall $R e$ height profile (i.e. the turbulent diffusivity) by 10 results in lifting the turbopause by approximately $3 \mathrm{~km}$. See the Discussion for further explanation 
$\mathbf{U}_{\perp} \approx \mathbf{V}_{\perp}-\frac{1}{v_{\text {in }}} \frac{B e}{m_{i}} \frac{\mathbf{E}}{|\mathbf{B}|}$

$\mathbf{U}_{\perp} \approx \mathbf{V}_{\perp}-\frac{e}{m_{i}} \frac{\mathbf{E}}{v_{i n}}$

where $m_{i}$ is the ion mass (note $\mathbf{U}_{\perp}, \mathbf{V}_{\perp}$ and $\mathbf{E}$ are vectors).

Typically: $v_{i n}=2.6 \times 10^{-15}\left(n_{n}+n_{i}\right) M_{n}^{-1 / 2}$ per second, $M_{n}=30.5 \mathrm{amu}$, the neutral molecular weight, $n_{n}$ and $n_{i}$ are the neutral and ion number densities respectively, $e=1.6 \times 10^{-19}$ coulomb, the electronic charge, and $m_{i}=30 \times 1.67 \times 10^{-27} \mathrm{~kg}$.

As explained by Fujii et al. (1998) we are able to determine the neutral wind as a function of height. Thus, we may estimate the gradient Richardson Number Ri:

$R i=\frac{\omega_{B}^{2}}{\left(\frac{\mathrm{dU}}{\mathrm{d} z}\right)^{2}}$

where the Brunt-Väisälä frequency $\omega_{B}$ is given by:

$\omega_{B}^{2}=\frac{g}{T}\left[\frac{\mathrm{d} T}{\mathrm{~d} z}+\frac{g}{C_{p}}\right]$

wherein $g$ is the acceleration due to gravity, $T$ is the temperature, $C_{p}$ is the specific heat at constant pressure and $g / C_{p}$ may be taken as $0.0098 \mathrm{~km}^{-1}$. Profiles of $T$ may be obtained from models (e.g. Hedin, 1991) allowing us to search for the instability condition $R i \leq 0.25$ as a function of height. Using incoherent scatter radar, Nozawa and Brekke (1999) have reported neutral shears exceeding $20 \mathrm{~ms}^{-1} \mathrm{~km}^{-1}$ in the lower thermosphere, which also corresponds to the typical value of $2 \times 10^{-2} \mathrm{rad} \mathrm{s}^{-1}$ for the Brunt-Väisälä frequency. Since these are presented as averages over several days of observation, it is reasonable to suppose that even higher shears may be present on occasion. The value $20 \mathrm{~ms}^{-1} \mathrm{~km}^{-1}$ corresponds to $R i \approx 1$. Although $R i \leq 0.25$ is a necessary but not sufficient condition for creation of turbulence, 0.4 would suffice if turbulence were already present.

Next, recall the Reynolds number $R e$; the equivalent metric for ion-drag induced mixing could thus be:

$\aleph \equiv \frac{\text { (ion-drag induced neutral wind) } \times \text { length }}{\text { kinematic viscosity }}$

However, another formalism of $R e$ utilises the reciprocals of the characteristic timescales for the inertial and viscous controlled dynamics (e.g. Tennekes and Lumley, 1972) (or in other words turbulent and molecular mixing time constants) such that an alternative to Eq. (5a) might be:

$\aleph \equiv \frac{1 / T_{\text {electrodynamic }}}{1 / T_{\text {molecular }}}$

which represents the ratio between ion-drag induced mixing $\left(T_{\text {electrodynamic }}\right)$ and viscosity $\left(T_{\text {molecular }}\right)$. From Tennekes and Lumley (1972) we see that

$T_{\text {molecular }}=L_{B}^{2} / v$ where $v$ is the kinematic viscosity (not to be confused with a collision frequency). In order to test this approach, let $T_{\text {electrodynamic }}$ be simply given by the reciprocal of the neutral-ion collision frequency $v_{n i}=v_{i n} \cdot n_{i} / n_{n}$ where $n_{i}$ and $n_{n}$ are the ion and neutral number densities respectively (e.g. Ratcliffe, 1972). In the same way that $R e$ is evaluated in a variety of ways, the analogous metric may also be; it is our intention that Eq. (5a) should be taken as the definition.

The metric $\aleph$ is required to be greater than unity for ion-drag mixing to dominate molecular diffusive mixing. Thus for neutral gas turbulence instigation (by iondrag):

$\aleph=\left(\frac{2.6 \cdot 10^{-15} \cdot n_{i}}{\sqrt{M_{n}}} \cdot \frac{L_{B}^{2}}{v}\right) \geq 1 \quad$ AND $\quad R i \leq 0.25$

In Eq. (7), we see that we combine a condition that iondrag induced neutral dynamics must dominate over viscous damping: $\aleph \geq 1$, and that $R i \leq 0.25$. Typical iondensity profiles may be obtained from models such as International Reference Ionosphere (IRI) (e.g. Bilitza et al., 1993), however, under conditions of auroral precipitation, the night-time E-region peak density may easily exceed the day-time value by an order of magnitude (e.g. Brekke, 1997). A typical ion density profile has been selected at random (midday, solstice $70^{\circ} \mathrm{N}$ ) from IRI and multiplied by 10 to simulate disturbed conditions (E-region peak was $1.4 \times 10^{12}$ electrons $\mathrm{m}^{-3}$ for the disturbed case). Similarly MSIS (Hedin, 1991) has been used as the basis for obtaining the corresponding profile of kinematic viscosity. The profiles of $T_{\text {electrodynamics }}$ and $T_{\text {molecular }}$ (using $L_{B}=200 \mathrm{~m}$ ) that result are shown in Fig. 3. The molecular process exhibits an exponential decrease of timescale with

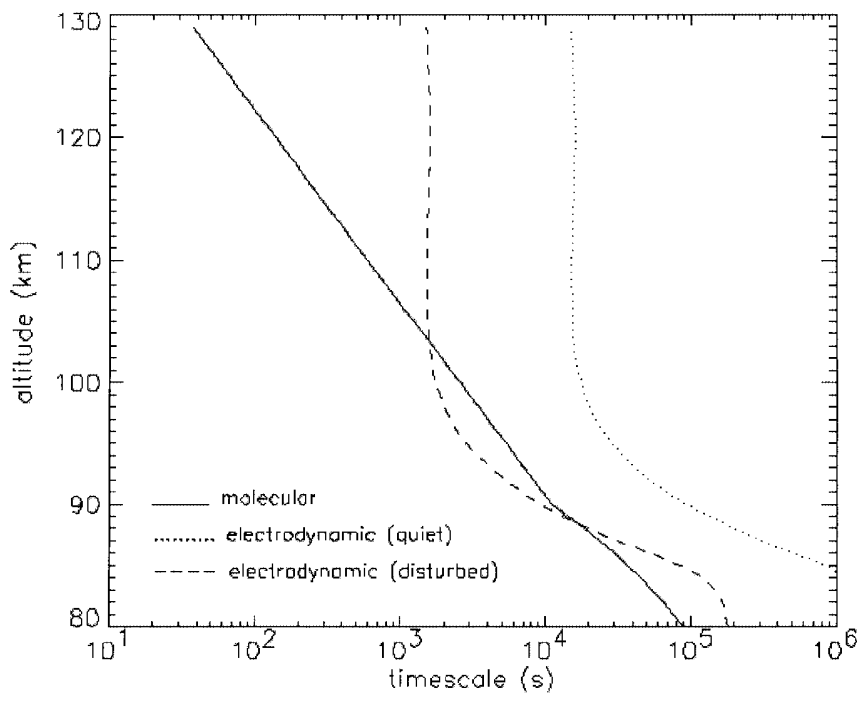

Fig. 3. Time constants for processes in the atmosphere. Solid line: molecular diffusion time scale defined as the square of a length scale, in this case we use the buoyancy length scale, $L_{B}$, estimated to be $200 \mathrm{~m}$, divided by the kinematic viscosity. Dashed and dotted lines are the corresponding time scales for ion-drag for disturbed and quiet conditions respectively. $L_{B}$ is chosen because we shall compare these time scales for identifying potential turbulent flows 


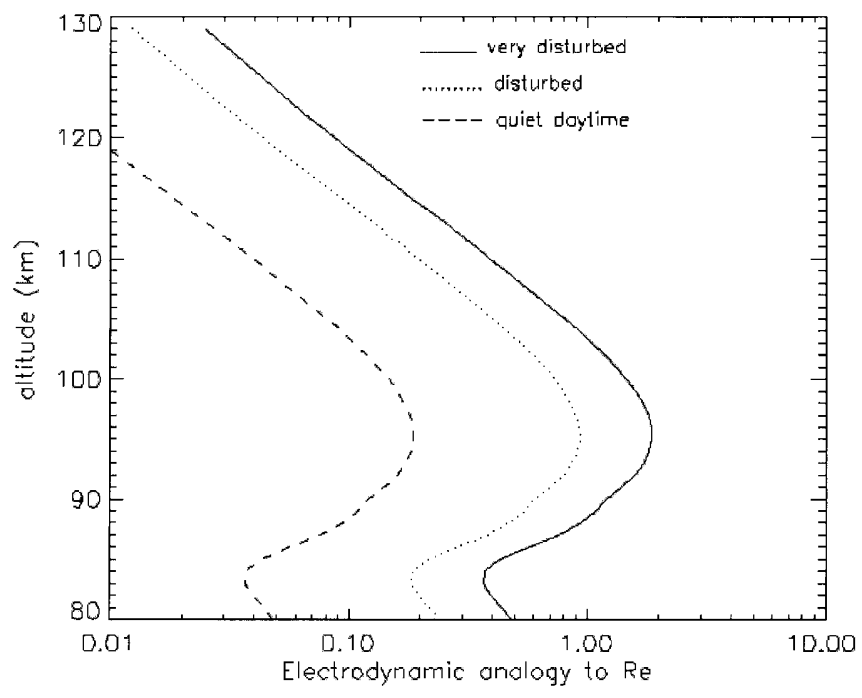

Fig. 4. Ratio of viscous (molecular diffusion) time scale to time between neutral-ion collisions, as a function of height for various degrees of ionisation. An E-region peak of $1.4 \times 10^{12}{\text { electrons } \mathrm{m}^{-3}}^{-3}$ causes the ratio, the electrodynamics analogy to the Reynolds Number, proposed here, to exceed unity. For the very disturbed case (e.g. auroral precipitation) viscous "stabilisation" dominates above $105 \mathrm{~km}$; ion-drag "destabilisation" is present between 88 and $103 \mathrm{~km}$, and ion-drag is less significant below $88 \mathrm{~km}$ due to low ion density. Real ionisation profiles, containing, for example, sporadic-E layers, might well result in multiple thin layers of high "electrodynamics Reynolds Number"

height, while that of the electrodynamics is more complicated. Between 88 and $103 \mathrm{~km}$, during disturbed conditions, the neutral atmosphere responds faster to the ion motion than to its own viscous damping. The ratio of these time scales, $T_{\text {molecular }} / T_{\text {electrodynamics, }}$, the suggestion for the proposed analogy to $R e$, is plotted in Fig. 4. for E-region peaks of $1.4 \times 10^{11}, 7 \times 10^{11}$ and $1.4 \times 10^{12}$ electrons $\mathrm{m}^{-3}$. The reduction towards lower heights is due to the rapid decrease of ion-density, whereas the fall-off with height above $100 \mathrm{~km}$ is due to the increasing neutral kinematic viscosity. In a height region around $95 \mathrm{~km}$, ion-drag is more important for the neutral motion than neutral viscosity. Of course, this height regime may be more complex in reality and may appear at different altitudes depending on the nature of the ionisation. Recall that the background atmosphere and ionosphere used to generate these two figures are from models (in fact for summer) and so the actual values will certainly vary somewhat. Furthermore we have selected a turbulent buoyancy length scale $L_{B}$ of $200 \mathrm{~m}$ in this example.

\section{Conclusion}

A mechanism is suggested to explain the observational result of Danilov et al. (1979): that the turbopause height depends in some way on geomagnetic activity. Disturbed geomagnetic conditions characterised by values of the local linear index $a_{k}$ of around 100 can probably coincide with a "lifting" of the turbopause by some $15 \mathrm{~km}$. By definition of the turbopause, turbulence is not possible above this height; however, in the scenario described here, turbulence is forced by electrodynamics at altitudes where it is normally damped by viscosity. We hypothesise that the cause of the DKP79 result lies in auroral activity, which in turn causes anomlous forcing of neutral turbulence in the lower thermosphere at height where turbulence is normally damped out. Several mechanisms may be proposed relating geomagnetic index and turbopause height, and we tentatively suggest that powerful and vertically limited ion drag can increase neutral dynamic instability occurrence in the lower thermosphere and thus induce enhanced neutral turbulence. Attempting to quantify such an effect, we propose a metric analogous to the Reynolds Number that indicates the relative importance of ion-drag on neutral motion to that of kinematic viscosity. One formulation of this metric might be $\aleph \equiv v_{n i} \cdot \frac{L_{B}^{2}}{v}=\frac{2.6 \cdot 10^{-15} n_{i}}{\sqrt{M_{n}}} \cdot \frac{L_{B}^{2}}{v}$ this representing the ratio between the timescale for molecular diffusion and that for neutral-ion collisions. The geomagnetic activity is principally due to E-region current systems which in turn depend on the nature of the electron (or positive ion) density profile $n_{i}(z)$.

The height of the turbopause is of interest because it indicates lower thermosphere turbulence activity and also information on turbulent mixing efficiency. The higher the turbopause, the higher up turbulence may redistribute species such as methane (important for water chemistry). Similarly, molecular oxygen is dissociated in the ionosphere; if the turbopause is located within the atomic oxygen production regime, the atomic oxygen may then be transported downwards enabling it to affect the ozone chemistry in the upper mesosphere. Thus, geomagnetic disturbances originating in electrodynamics may be capable of affecting vertical transport of species at high latitude, which in turn dictate phenomena such as noctilucent clouds and affect, for example, ozone content.

Acknowledgements. The author wishes to thank the referees of this work. Thanks also go to Asgeir Brekke and Enok Palm.

Topical Editor D. Murtagh thanks T. A. Blix and R. Roper for their help in evaluating this paper.

\section{References}

Bilitza, D., K. Rawer, L. Bossy, and T. Gulyaeva, International Reference Ionosphere - past, present, future, Adv. Space Res. 13, 3-23, 1993.

Blum, P. W., and K. G. H. Schuchardt, Semi-theoretical global models of the eddy diffusion coefficient based on satellite data, J. Atmos. Terr. Phys., 40, 1137-1142, 1978.

Brekke, A., Physics of the upper polar atmosphere, Wiley, New York, 1997.

Danilov, A. D., and U. A. Kalgin, Eddy diffusion studies in the lower thermosphere, Adv. Space Res., 17, (11)17-(11)24, 1996.

Danilov, A. D., U. A. Kalgin, and A. A. Pokhunov, Variation of the mesopause level in polar regions, Space Res. XIX, 83, 173-176, 1979.

Fujii, R., S. Nozawa, S. C. Buchart, N. Matuura, and A. Brekke, The motion of ions in the auroral ionosphere, J. Geophys. Res., 103, 20 685-20 695, 1998. 
Hall, C. M., A. Brekke, O. V. Martynenko, and A. A. Namgaladze, Turbulent energy dissipation in the high-latitude mesosphere: the PGI97 model, J. Atmos Solar. Terr. Phys., 60, 331-336, 1998.

Hedin, A. E., Extension of the MSIS thermosphere model into the middle and lower atmosphere, J. Geophys. Res., 96, 1159-1172, 1991.

Jacobs, J. A., Geomagnetism volume 3, Academic Press, London, 1989.

Nozawa, S., and A. Brekke, Seasonal variation of the auroral E-region neutral wind for different solar activities, J. Atmos. Solar-Terr Phys., 61, 585-605, 1999.
Ratcliffe, J. A., An introduction to the ionosphere and magnetosphere, Cambridge University Press, Cambridge, 1972.

Roper, R. G., and J. W. Brosnahan, Imaging Doppler interferometry and the measurement of atmospheric turbulence, Radio Sci., 32, 1137-1148, 1997.

Shimazaki, T., Effective eddy diffusion coefficient and atmospheric composition in the lower thermosphere. J. Atmos. Terr. Phys., 33, 1383-1401, 1971

Tennekes, H., and J. L. Lumley, A first course in turbulence, MIT, USA., 1972.

Weinstock, J., Vertical turbulent diffusion in a stably stratified fluid, J. Atmos. Sci., 35, 1022-1027, 1978. 